\title{
Cusum charts for preliminary analysis of individual observations
}

\author{
Alex J. Koning* \\ Econometric Institute \\ Erasmus University Rotterdam \\ P.O. Box 1738 \\ NL-3000 DR Rotterdam \\ koning@few.eur.nl
}

\author{
Ronald J.M.M. Does ${ }^{\dagger}$ \\ Institute for Business and Industrial Statistics \\ University of Amsterdam \\ Plantage Muidergracht 24 \\ NL-1018 TV Amsterdam \\ The Netherlands \\ rjmmdoes@wins.uva.nl
}

June 16, 1997

\begin{abstract}
A preliminary Cusum chart based on individual observations is developed from the uniformly most powerful test for the detection of linear trends. This Cusum chart is compared with several of its competitors which are based on the likelihood ratio test and on transformations of standardized recursive residuals on which for instance the Q-chart methodology is based. It turns out that the new proposed Cusum chart is not only superior in the detection of linear trend outof-control conditions, but also in the detection of other out-of-control situations. Approximate control limits, determined from simulation, and an example of its use in practice are given for the proposed Cusum chart.
\end{abstract}

\section{Contents}

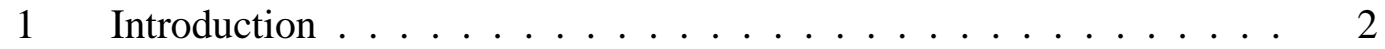

2 Recursive residuals and $Q$-statistics ............... 3

3 A new Cusum chart .................. 5

4 A practical example ..................... 9

5 A comparison of the methods . . . . . . . . . . . . . 10

A Appendix: a generalized Cusum chart . . . . . . . . . . . . 14

${ }^{*}$ Dr. Koning is an Assistant Professor in the Econometric Institute. He is a Member of ASQC.

${ }^{\dagger}$ Dr. Does is a Professor in the Department of Mathematics and Director of the Institute for Business and Industrial Statistics. He is a Member of ASQC. 


\section{Introduction}

Control charts are basic and powerful tools in Statistical Process Control [SPC] and are widely accepted and applied techniques for controlling various industrial processes. Originated by Shewhart in 1924 [cf. Shewhart (1931)], the effectiveness of control charts is due in part to their simplicity. In fact these charts are simple graphs with time on the horizontal axis and a quality characteristic such as sample mean or sample range plotted on the vertical axis. Shewhart developed the use of 3-sigma control limits as action limits, i.e. if the quality characteristic is outside these limits then the process is called out-of-control and action is needed to eliminate the special cause.

Another technique to control an industrial process was developed by Page (1954). He proposed the so-called Cumulative-sum [Cusum] chart. This technique plots the cumulative sums of deviations of the sample values of a quality characteristic from a target value against time. There are two ways to represent Cusums, the tabular [or algorithmic] Cusum, and the V-mask form of the Cusum.

It is said [cf. Montgomery (1996)] that Shewhart-type control charts for averages are very effective if the magnitude of the shift is 1.5-sigma to 2-sigma or larger. For smaller shifts, the Cusum is a good alternative or additional tests for special causes [cf. Nelson (1984)] are needed to improve the effectiveness of the Shewhart-type control chart.

In the literature two situations of using control charts are distinguished: stage 1 [retrospective] and stage 2 [prospective]. In stage 1 historical data are analyzed to decide if the process is in statistical control and to estimate the in-control parameters of the process. The next stage, the so-called prospective stage 2 , is started when the analysis of past data did not reveal any out-of-control signals. It is very important that all special causes are detected in stage 1 because this leads to a better understanding of the process and it avoids inflation of the estimates of the parameters needed for stage 2 . In course of time the preliminary estimates are revised based on available data from stage 2 , and again we are in a stage 1 situation. This updating and checking of the parameters is a recurrent phenomena, but one hopes to move into a situation where out-of-control signals are fairly rare.

Today's manufacturing environment hardly resembles the high volume production of the twenties to the early eighties, in which period SPC charting methods were introduced. Current manufacturing practice is typically characterized by either frequent set up changes to accommodate a wide range of different products [short run processes], or intrinsically low production volumes of the same type of product. Printed circuit boards for specialized applications are an example of the first situation, the production of wafer steppers for semiconductor production is an example of the last. The last few years there is a revival of interest to handle these problems. The increasing use of computers for SPC applications enables industrial statisticians to consider other approaches from mathematical statistics that are more powerful in discovering special causes.

Quesenberry $(1991,1995)$ introduced $Q$-charts and Del Castillo and Montgomery 
(1994) developed some enhancements and alternative methods. All these methods require the use of a computer. The present study was motivated by a recent paper of Sullivan and Woodall (1996), where a preliminary control chart for individual observations based on a likelihood ratio test was developed; this chart cannot be applied in a stage 2 situation, and hence is exclusively intended for the stage 1 situation. It is remarkable that some of the new proposed charts have been based on well-known papers in econometrics. For instance the $Q$-chart methodology can be written as a transformation of standardized recursive residuals as proposed in section 2.3 of Brown, Durbin and Evans (1975). Also the likelihood ratio test [LRT] control chart in Sullivan and Woodall (1996) is strongly related to Quandt's log-likelihood ratio technique [cf. Quandt (1960)].

Hence, in this paper we consider individual observations and we focus on the retrospective [stage 1] situation. The structure of the paper is as follows. After introducing standardized recursive residuals and Q-statistics, we develop a new Cusum-type chart for the detection of linear trends based on the uniformly most powerful test, and compare this new chart with the LRT chart of Sullivan and Woodall (1996), the Cusum chart of Brown, Durbin and Evans (1975); it turns out that the proposed Cusum chart has the best properties to detect trends and shifts in the data. In the appendix a generalisation of the proposed chart is given.

\section{Recursive residuals and $Q$-statistics}

In recent years many control charts have been suggested which build on the $Q$-statistics proposed in Quesenberry (1991). In this section we show how results concerning recursive residuals [well-known in econometrics] can be used to give a complete description of the joint distribution of $Q$-statistics.

Consider the linear model

$$
X_{i}=z_{i}^{\prime} \beta+\epsilon_{i}, \quad i=1,2, \ldots
$$

where the $\epsilon_{i}$ 's are independent normal random variables with expectation zero and variance $\sigma^{2}$, and $\beta$ is an unknown $p$-dimensional vector. Let $Z_{i}$ denote the $i \times p$ matrix with rows $z_{1}^{\prime}, z_{2}^{\prime}, \ldots, z_{i}^{\prime}$, and let

$$
\hat{\beta}_{i}=\left(Z_{i}^{\prime} Z_{i}\right)^{-1} Z_{i}\left(\begin{array}{c}
X_{1} \\
\vdots \\
X_{i}
\end{array}\right)
$$

be the least squares estimator of $\beta$ at time $i$. Brown, Durbin and Evans (1975) introduced the standardized recursive residual

$$
Y_{i}=\frac{X_{i}-z_{i}^{\prime} \hat{\beta}_{i-1}}{\sqrt{1+z_{i}^{\prime}\left(Z_{i-1}^{\prime} Z_{i-1}\right)^{-1} z_{i}}}, \quad i=p+1, p+2, \ldots,
$$


and showed that the random variables $Y_{p+1}, Y_{p+2}, \ldots$ are independent normal variables with expectation zero and variance $\sigma^{2}$.

In practical applications $\sigma^{2}$ typically is unknown, and needs to be estimated. At time $i$ the estimator

$$
S_{i}^{2}=\frac{1}{i-p} \sum_{j=1}^{i}\left(X_{j}-z_{j}^{\prime} \hat{\beta}_{i}\right)^{2}
$$

can be used. In section 2.3 of Brown, Durbin and Evans (1975) a cumulative sum chart is proposed which applies a V-mask procedure to the cumulative sum

$$
\frac{1}{S_{n}} \sum_{j=1}^{i} Y_{j}
$$

with $Y_{j}$ and $S_{n}$ given by (1) and (2). We shall refer to this chart as the BDE Cusum chart.

In Lemma 2 of Brown, Durbin and Evans (1975) the recursive formula

$$
(i-p) S_{i}^{2}=(i-p-1) S_{i-1}^{2}+Y_{i}^{2}
$$

is given, which implies that $S_{i-1}^{2}$ only depends on $Y_{p+1}, \ldots, Y_{i-1}$, and hence is independent of $Y_{i}$. Since $(i-p-1) S_{i-1}^{2} / \sigma^{2}$ has a $\chi^{2}$-distribution with $i-p-1$ degrees of freedom, it follows by standard statistical theory that $Y_{i} / S_{i-1}$ has a $t$-distribution with the same degrees of freedom for $i \geq p+2$.

We now show that $Y_{3} / S_{2}, \ldots, Y_{n} / S_{n-1}$ are independent. First, we show that $Y_{i+1} / S_{i}$ and $Y_{i+2} / S_{i+1}$ are independent: since $S_{i+1}$ is a complete and sufficient statistic for $\sigma$ if only $X_{1}, X_{2}, \ldots, X_{i+1}$ are observed, it follows from the fact that the distribution of $Y_{i+1} / S_{i}$ does not depend on $\sigma^{2}$ that $Y_{i+1} / S_{i}$ and $S_{i+1}$ are independent; moreover, since $Y_{3} / S_{2}, \ldots, Y_{i} / S_{i-1}$ and $S_{i}$ may considered to be functions of $Y_{2}, \ldots, Y_{i}$, it immediately follows from the independence between $Y_{2}, \ldots, Y_{i}$ and $Y_{i+1}$ that $Y_{i} / S_{i-1}$ and $Y_{i+1} / S_{i}$ are independent. The general statement follows by an induction argument.

In the special case where $z_{i}$ is equal to the scalar 1 for every $i$, we have that $p$ is equal to 1 , and $z_{i}^{\prime} \hat{\beta}_{i-1}$ is equal to $\bar{X}_{i-1}$, the mean of $X_{1}, \ldots, X_{i-1}$; it follows that (1) and (2) specialize to

$$
\begin{gathered}
Y_{i}=\frac{X_{i}-\bar{X}_{i-1}}{\sqrt{1+(i-1)^{-1}}}=\sqrt{\frac{i-1}{i}}\left(X_{i}-\bar{X}_{i-1}\right), i=2,3, \ldots, \\
S_{i}^{2}=\frac{1}{i-1} \sum_{j=1}^{i}\left(X_{j}-\bar{X}_{j-1}\right)^{2} .
\end{gathered}
$$

Transforming the ratio $Y_{i} / S_{i-1}$, we obtain the following statistics

$$
Q_{i}=\Phi^{-1}\left(G_{i-p-1}\left(\frac{Y_{i}}{S_{i-1}}\right)\right),
$$


which were called $Q$-statistics in Quesenberry (1991). Here $\Phi^{-1}$ denotes the inverse of the standard normal cumulative distribution function, and $G_{\nu}$ denotes the $t$-cumulative distribution function with $\nu$ degrees of freedom. It immediately follows from our description of the joint distribution of $Y_{3} / S_{2}, Y_{4} / S_{3}, \ldots$ that $Q_{3}, Q_{4}, \ldots$ are independent standard normal random variables.

In the remainder of this paper the special case $z_{i}=1$ for all $i$ will be used as a model for the process under in-control conditions.

\section{A new Cusum chart}

In this section we propose a Cusum chart which to the best of our knowledge did not appear in literature before. The proposed Cusum chart originates from exploring the behavior of $Y_{i}$ as defined by (3) under the linear model

$$
X_{i}=\mu+i \theta+\epsilon_{i}, \quad i=1,2, \ldots
$$

where the $\epsilon_{i}$ 's are independent normal random variables with expectation zero and unknown variance $\sigma^{2}$. In Appendix A a more general model is considered. We may write

$$
Y_{i}=\sqrt{\frac{i-1}{i}}\left(i-\frac{1}{i-1} \sum_{j=1}^{i-1} j\right) \theta+\delta_{i}=\frac{\theta}{2} \sqrt{i(i-1)}+\delta_{i},
$$

where

$$
\delta_{i}=\sqrt{\frac{i-1}{i}}\left(\epsilon_{i}-\frac{1}{i-1} \sum_{j=1}^{i-1} \epsilon_{j}\right) .
$$

Observe that $\delta_{2}, \ldots, \delta_{n}$ are independent normal random variables with expectation zero and common variance $\sigma^{2}$, whereas $\delta_{1}$ is degenerate in zero.

Let us for the moment suppose that $\sigma^{2}$ is known. The joint density of $Y_{2}, \ldots, Y_{i}$ is given by

$$
\left(2 \pi \sigma^{2}\right)^{-(i-1) / 2} \exp \left\{-\frac{1}{2 \sigma^{2}}\left(\sum_{j=2}^{i} y_{j}^{2}-\theta \sum_{j=2}^{i} y_{j} \sqrt{j(j-1)}+\frac{\theta^{2}}{4} \sum_{j=2}^{i} j(j-1)\right)\right\},
$$

which can be rewritten in the form

$$
h\left(y_{2}, \ldots, y_{i}\right) k(\theta) \exp \left\{U\left(y_{2}, \ldots, y_{i}\right) c(\theta)\right\},
$$

where

$$
c(\theta)=\frac{\theta}{2 \sigma^{2}}
$$

is an increasing function of the parameter $\theta$. It follows [cf. Lehmann (1994), Corollary 3.2, p. 80] that the joint density of $Y_{2}, \ldots, Y_{i}$ has a monotone likelihood ratio in

$$
U_{i}=U\left(Y_{2}, \ldots, Y_{i}\right)=\sum_{j=2}^{i} Y_{j} \sqrt{j(j-1)}
$$


with respect to $\theta$. Thus, if $Y_{2}, \ldots, Y_{i}$ are observed, then the one-sided test based on $U_{i}$ is uniformly most powerful for $\theta$ [cf. Lehmann (1994), Theorem 2(i), p.78]. This suggests that charts for detecting linear trend should be constructed by cumulating $Y_{j} \sqrt{j(j-1)}$ rather than $Y_{j}$ itself.

Observe that $U_{i}$ has expectation $\theta \sum_{j=2}^{i} j(j-1)$ and variance $\sigma^{2} \sum_{j=2}^{i} j(j-1)$. Thus, both the expectation and the variance of $U_{i}$ are linear in $\sum_{j=2}^{i} j(j-1)$, which indicates that plotting $U_{i}$ versus $\sum_{j=2}^{i} j(j-1)$ is preferable over plotting $U_{i}$ versus $i$. Moreover, since a nonzero value of $\theta$ corresponds to a linear trend in the plot of $U_{i}$ versus $\sum_{j=2}^{i} j(j-1)$, applying a V-mask procedure to this plot seems reasonable.

It was shown in Lucas (1982) [cf. Montgomery (1996)] that a V-mask cumulative sum chart may be represented by means of a pair of so-called tabular cumulative sums. Following the same line of reasoning, one may show that the $\mathrm{V}$-mask procedure applied to the plot of $U_{i}$ versus $\sum_{j=2}^{i} j(j-1)$ is equivalent to imposing a control limit $h$ on the pair of one-sided cumulative sums $S_{H, i}$ and $S_{L, i}$ defined by

$$
\begin{aligned}
& S_{H, i}=\max \left(0, S_{H, i-1}+\sqrt{i(i-1)}\left(Y_{i}-f \sqrt{i(i-1)}\right)\right), \\
& S_{L, i}=\max \left(0, S_{L, i-1}+\sqrt{i(i-1)}\left(-Y_{i}-f \sqrt{i(i-1)}\right)\right),
\end{aligned}
$$

where $f$ is the so-called reference value. The one-sided cumulative sums $S_{H, i}$ and $S_{L, i}$ share their time-scale with $U_{i}$; that is, they should be plotted versus $\sum_{j=2}^{i} j(j-1)$ rather than $i$.

The charts just described distinguish themselves from the classical cumulative sum charts by employing a transformed time-scale. Although time-transformations are seldom used in statistical process control, they are being used in other industrial applications of statistics. For example, the Total Time on Test plot in reliability [cf. Barlow and Campo (1975)].

A disturbing effect of the time-transformation is that the time instance $\sum_{j=2}^{n} j(j-$ 1) at which the "last" observation in the sample is observed, does not depend linearly on the sample size anymore. This can be repaired by introducing

$$
b_{n}=\sqrt{\frac{3}{n(n+1)}}
$$

and plotting $b_{n} U_{i}$ versus $b_{n}^{2} \sum_{j=2}^{n} j(j-1)$ rather than plotting $U_{i}$ versus $\sum_{j=2}^{n} j(j-1)$ [observe that the variance of $b_{n} U_{n}$ is given by $\sigma^{2}(n-1)$ ]. Equivalently, one may plot the tabular Cusums $b_{n} S_{L, i}$ and $b_{n} S_{H, i}$ versus $b_{n}^{2} \sum_{j=2}^{n} j(j-1)$.

Until now, we have only considered the situation where the variance $\sigma^{2}$ is known. When $\sigma^{2}$ is unknown, a problem occurs due to the dependence of the control limits on $\sigma^{2}$. However, this dependence is readily removed by dividing $b_{n} U_{i}$ [or equivalently, by dividing the the tabular Cusums $b_{n} S_{L, i}$ and $b_{n} S_{H, i}$ ] by the estimator $S_{n}$ as defined in (4). 
Let $h_{\alpha}$ denote the control limit for the tabular Cusums $b_{n} S_{L, i} / S_{n}$ and $b_{n} S_{H, i} / S_{n}$ which yields an overall in-control signalling probability $\alpha$. In Table 1 values of $h_{\alpha}$ are given for $f=0$ and various choices of $\alpha$ and $n$. These values are based on 10,000 simulations, which may yield inaccurate results for very small $\alpha$. Our adherence to $f=0$ will be explained later, in the discussion of our simulation results.

\begin{tabular}{|c|cccc|}
\hline$n$ & \multicolumn{4}{|c|}{ Control limits } \\
& $h_{0.001}$ & $h_{0.005}$ & $h_{0.01}$ & $h_{0.05}$ \\
\hline 5 & 3.97 & 3.89 & 3.84 & 3.56 \\
10 & 7.96 & 7.32 & 7.03 & 6.05 \\
15 & 10.78 & 9.98 & 9.46 & 7.93 \\
20 & 13.61 & 12.27 & 11.54 & 9.50 \\
25 & 15.62 & 13.93 & 13.10 & 10.79 \\
30 & 17.08 & 15.49 & 14.52 & 11.93 \\
35 & 19.18 & 16.79 & 15.75 & 13.24 \\
40 & 19.94 & 18.16 & 17.20 & 14.01 \\
45 & 21.96 & 19.48 & 18.48 & 15.06 \\
50 & 23.17 & 21.04 & 19.36 & 16.03 \\
60 & 26.58 & 23.10 & 21.72 & 17.66 \\
70 & 28.89 & 24.89 & 23.02 & 19.08 \\
80 & 31.75 & 26.04 & 25.02 & 20.74 \\
90 & 32.09 & 28.78 & 26.94 & 22.25 \\
\hline
\end{tabular}

Table 1: Simulated control limits $h_{\alpha}$ for tabular Cusums $b_{n} S_{L, i} / S_{n}$ and $b_{n} S_{H, i} / S_{n}$ with $f=0$, resulting in an overall in-control signalling probability $\alpha=0.001,0.005,0.01,0.05$. Control limits are based on 10,000 simulations.

For $n$ sufficiently large, theoretical considerations based on formula (11.12) in Billingsley (1968) yield that $h_{0.001}, h_{0.005}, h_{0.01}$ and $h_{0.05}$ may be approximated by $\sqrt{13.41 n}, \sqrt{10.41 n}, \sqrt{9.14 n}$ and $\sqrt{6.24 n}$, respectively. Plots of the values in Table 1 versus $n$ suggest the following refinements:

$$
\begin{aligned}
h_{0.001} & \approx \sqrt{13.41 n-19.41 \sqrt{n}}, \\
h_{0.005} & \approx \sqrt{10.41 n-13.35 \sqrt{n}}, \\
h_{0.01} & \approx \sqrt{9.14 n-11.34 \sqrt{n}}, \\
h_{0.05} & \approx \sqrt{6.24 n-7.87 \sqrt{n}},
\end{aligned}
$$

which may be used for $n \geq 50$. 


\begin{tabular}{|c|c|c|c|c|c|c|c|c|c|}
\hline \multirow[t]{2}{*}{$i$} & \multirow[t]{2}{*}{$X_{i}$} & \multirow[t]{2}{*}{$Y_{i}$} & \multicolumn{2}{|c|}{$\begin{array}{c}\text { Proposed } \\
\text { Cusum chart }\end{array}$} & \multicolumn{2}{|c|}{$\begin{array}{c}\text { Multiplied by } \\
b_{n} / S_{n}=0.04519\end{array}$} & \multicolumn{2}{|c|}{$\begin{array}{l}\text { BDE } \\
\text { chart }\end{array}$} & \multirow[t]{2}{*}{$\begin{array}{l}\text { LRT } \\
\text { chart }\end{array}$} \\
\hline & & & lower & upper & lower & upper & lower & upper & \\
\hline 1 & -.69 & 0.0000 & .00 & .00 & .0000 & .0000 & .0000 & .0000 & .0000 \\
\hline 2 & .56 & .8839 & .00 & 1.25 & .0000 & .0565 & .0000 & .7033 & 1.5212 \\
\hline 3 & -.96 & -.7308 & 1.79 & .00 & .0809 & .0000 & .5814 & .1218 & 2.7731 \\
\hline 4 & -.11 & .2194 & 1.03 & .76 & .0465 & .0343 & .4069 & .2964 & 4.2768 \\
\hline 5 & -.25 & .0447 & .83 & .96 & .0375 & .0434 & .3713 & .3320 & 6.2854 \\
\hline 6 & .45 & .6755 & .00 & 4.66 & .0000 & .2106 & .0000 & .8695 & 6.4847 \\
\hline 7 & -.26 & -.0864 & .56 & 4.10 & .0253 & .1853 & .0688 & .8007 & 8.6341 \\
\hline 8 & .68 & .8045 & .00 & 10.12 & .0000 & .4573 & .0000 & 1.4408 & 8.2412 \\
\hline 9 & .22 & .2758 & .00 & 12.46 & .0000 & .5631 & .0000 & 1.6602 & 9.9020 \\
\hline 10 & -2.10 & -1.9543 & 18.54 & .00 & .8378 & .0000 & 1.5550 & .1053 & 6.6637 \\
\hline 11 & .65 & .8543 & 9.58 & 8.96 & .4329 & .4049 & .8752 & .7850 & 6.5225 \\
\hline 12 & -1.49 & -1.2690 & 24.16 & .00 & 1.0918 & .0000 & 1.8850 & .0000 & 7.9783 \\
\hline 13 & -2.49 & -2.1281 & 50.74 & .00 & 2.2930 & .0000 & 3.5782 & .0000 & 11.8817 \\
\hline 14 & -1.11 & -.6404 & 59.38 & .00 & 2.6834 & .0000 & 4.0878 & .0000 & 16.4430 \\
\hline 15 & .23 & .6983 & 49.26 & 10.12 & 2.2261 & .4573 & 3.5321 & .5557 & 16.7887 \\
\hline 16 & 2.16 & 2.5220 & 10.19 & 49.19 & .4605 & 2.2229 & 1.5255 & 2.5623 & 12.0269 \\
\hline 17 & 1.95 & 2.1652 & .00 & 84.90 & .0000 & 3.8367 & .0000 & 4.2851 & 9.0592 \\
\hline 18 & 1.54 & 1.6430 & .00 & 113.64 & .0000 & 5.1355 & .0000 & 5.5924 & 6.9959 \\
\hline 19 & .67 & .7073 & .00 & 126.72 & .0000 & 5.7266 & .0000 & 6.1551 & 6.2337 \\
\hline 20 & 1.09 & 1.0804 & .00 & 147.78 & .0000 & 6.6783 & .0000 & 7.0147 & 4.9985 \\
\hline 21 & 1.37 & 1.3009 & .00 & 174.44 & .0000 & 7.8831 & .0000 & 8.0498 & 3.6331 \\
\hline 22 & .69 & .5760 & .00 & 186.82 & .0000 & 8.4426 & .0000 & 8.5081 & 3.2395 \\
\hline 23 & 2.26 & 2.0858 & .00 & 233.74 & .0000 & 10.5630 & .0000 & 10.1677 & 1.6164 \\
\hline 24 & 1.86 & 1.6055 & .00 & 271.46 & .0000 & 12.2676 & .0000 & 11.4452 & .7447 \\
\hline 25 & .62 & .3250 & .00 & 279.42 & .0000 & 12.6273 & .0000 & 11.7037 & .4149 \\
\hline 26 & -1.04 & -1.3155 & 33.54 & 245.88 & 1.5157 & 11.1116 & 1.0467 & 10.6570 & 2.4402 \\
\hline 27 & 2.30 & 2.0117 & .00 & 299.18 & .0000 & 13.5203 & .0000 & 12.2576 & 2.4140 \\
\hline 28 & .07 & -.2513 & 6.91 & 292.27 & .3123 & 13.2080 & .2000 & 12.0577 & 2.6496 \\
\hline 29 & 1.49 & 1.1528 & .00 & 325.12 & .0000 & 14.6925 & .0000 & 12.9749 & .0000 \\
\hline 30 & .52 & .1600 & .00 & 329.84 & .0000 & 14.9058 & .0000 & 13.1022 & .0000 \\
\hline
\end{tabular}

Table 2: Application to the data in Table 2 in Sullivan and Woodall (1996). The following charts are computed: the propsed Cusum chart [appropriately rescaled], the BDE Cusum chart in tabular form, and the LRT chart [using (1) in Sullivan and Woodall (1996)]. 


\section{A practical example}

In this section we illustrate by means of an example that the proposed Cusum chart is not only superior in the detection of linear trend out-of-control conditions, but is highly efficient in the detection of other out-of-control conditions as well. In the next section this shall be confirmed by means of a simulation study.

The example concerns data given in Table 2 in Sullivan and Woodall (1996). Thirty observations $X_{1}, \ldots X_{30}$ were generated from a standard normal distribution, and a value 1 was added to the last fifteen observations $X_{16}, \ldots, X_{30}$. Thus, the data exhibit a sudden shift in the middle of the sample.

Table 2 presents the data $X_{i}$, the recursive residuals $Y_{i}$, the tabular Cusums $S_{L, i}$ and $S_{H, i}$, the appropriately rescaled tabular Cusums $b_{n} S_{L, i} / S_{n}$ and $b_{n} S_{H, i} / S_{n}$ [observe that 0.04519 is the ratio of $b_{n}=0.05680$ and $S_{n}=1.2568$ ], the BDE Cusum chart in tabular form, and the LRT chart.

The upper tabular Cusum $b_{n} S_{H, i} / S_{n}$ takes a maximal value 14.9058 , which is beyond 14.52, the value of $h_{0.01}$ for $n=30$ as given by Table 1 . This indicates the existence of a sudden shift. Moreover, observe that this maximal value starts to build up after the $15^{\text {th }}$ observation, which indicates the location of a special cause.

The tabular Cusums $b_{n} S_{L, i} / S_{n}$ and $b_{n} S_{H, i} / S_{n}$ were designed to have comparable in-control behavior as the BDE tabular Cusums. Thus, for the BDE Cusums the control limits in Table 1 roughly hold. The upper BDE Cusum takes a maximal value 13.1022, somewhere between $h_{0.01}$ and $h_{0.05}$.

In contrast, the LRT chart has rather different behavior. Its maximal value is 16.7887 , which is close to 16.09 , a control limit which yields a 5 percent in-control overall signalling probability for the LRT chart [obtained by simulation]. The location of the maximal value of the LRT chart [in this case $i=15$ ] is the maximum likelihood estimator of the location of the sudden shift.

Graphical representations of the charts in Table 2 are found in Figure 1. In addition, Figure 1 represents the Cusum and Shewhart charts from Quesenberry (1995) based on $Q$-statistics [respectively referred to as the $Q$-Cusum and the $Q$-chart].

The $Q$-Cusum chart is rather similar to the BDE Cusum chart, especially later in the sample. Of course, this is a consequence of the close relation between $Q$-statistics and recursive residuals. The upper tabular $Q$-Cusum takes a maximal value 13.1695, in the vicinity of the maximal value taken by the upper tabular BDE Cusum.

The $Q$-chart itself, a Shewhart chart based on $Q$-statistics, is not very efficient in detecting sudden shifts, as Figure 1 illustrates. The $Q$-chart takes values between 2.6288 and 2.2414, which is quite in accordance with the in-control behavior of the Q-chart. 

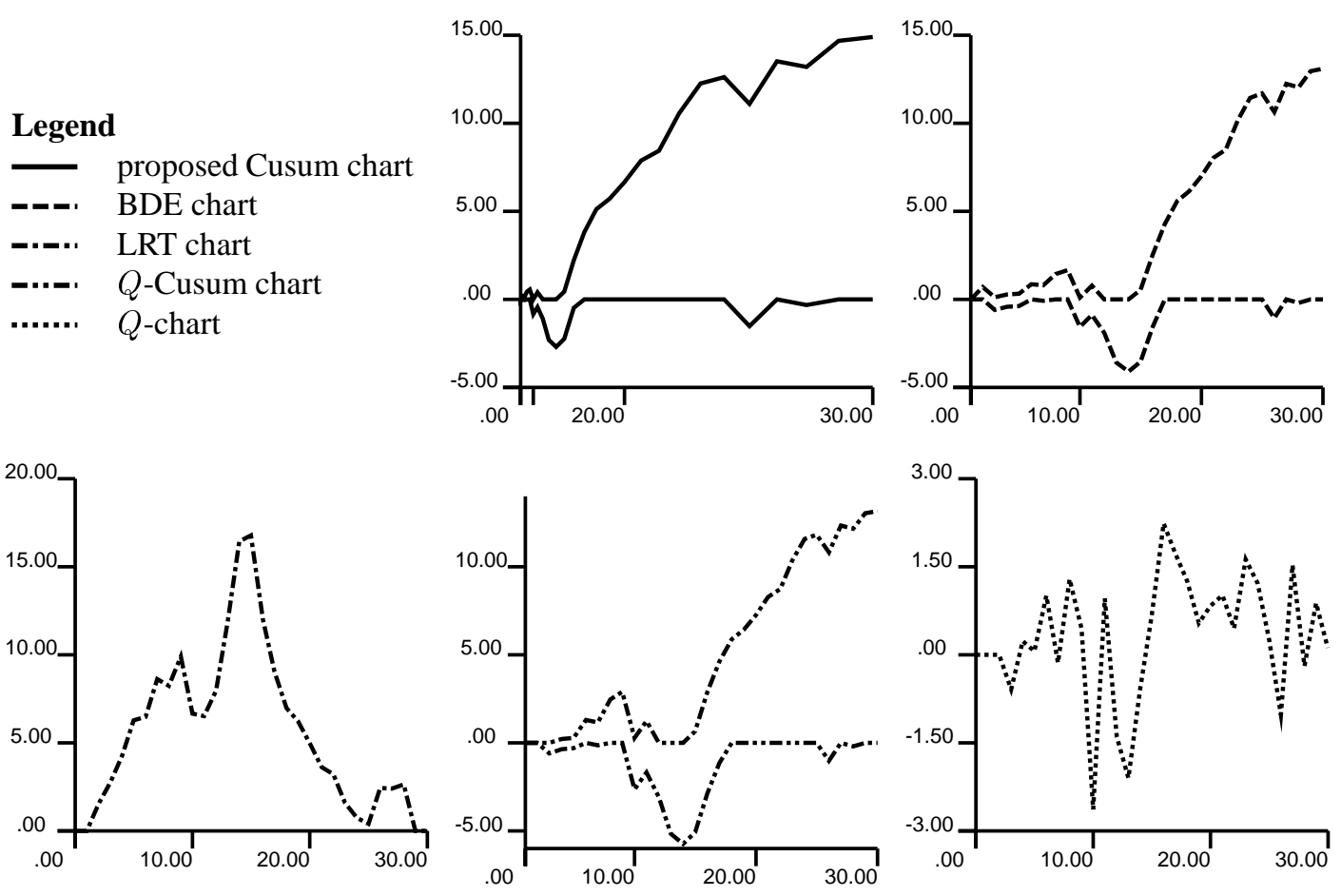

Figure 1: Charts of data from Table 2 in Sullivan and Woodall (1996). On the upper row the proposed Cusum chart and the BDE chart, on the lower row the likelihood ratio test chart, the $Q$-Cusum chart, and the $Q$-chart; in all Cusum charts we have taken $f$ equal to zero [cf. (6)]. According to our simulations the respective control limits $11.93,12.01,16.09,12.17$ and 3.12 yield a 5 percent in-control overall signalling probability

\section{A comparison of the methods}

In this section the proposed Cusum chart and several of its competitors are compared. Comparison will be made with the chart in section 2.3 of Brown, Durbin and Evans (1975), the LRT chart of Sullivan and Woodall (1996), and the Cusum and Shewhart charts from Quesenberry (1995) based on $Q$-statistics. Note that the traditional individual Shewhart chart based on the moving range is not included in the comparison because Sullivan and Woodall (1996) show that this chart was clearly inferior to the LRT chart.

Under six different out-of-control conditions 10,000 samples of size $n=6,12,18$, 24, 30 were simulated according to the model

$$
X_{i}=a_{i}+\epsilon_{i}, \quad i=1, \ldots, n,
$$


where the $\epsilon_{i}$ 's are independent standard normal random variables, and $a_{i}$ 's depend on the condition. Under the in-control condition all $a_{i}$ 's are equal to 0 .

Under each of the out-of-control conditions the deviance from in-control condition is indicated by the quantity

$$
\Delta\left(a_{1}, \ldots, a_{n}\right)=\sqrt{\sum_{i=1}^{n}\left(a_{i}-\bar{a}\right)^{2}},
$$

where $\bar{a}$ denotes the mean of $a_{1}, \ldots, a_{n}$. Below the structure of the $a_{i}$ 's in each of the six out-of control conditions is described.

Condition LTO The $a_{i}$ 's are linearly dependent on $i$. In this case a difference of $\sigma$ between $a_{1}$ and $a_{n}$ corresponds to the value $.3416, .3138, .3052, .3010, .2985$ of $\Delta\left(a_{1} \ldots, a_{n}\right)$ for $n=6,12,18,24,30$ respectively.

Condition LT1 The $a_{i}$ 's are constant up to relative position $1 / 3$ within the sample, and linearly dependent on $i$ from this position onwards. In this case a difference of $\sigma$ between $a_{1}$ and $a_{n}$ corresponds to the value $.3727, .3536, .3469, .3436, .3416$ of $\Delta\left(a_{1} \ldots, a_{n}\right)$ for $n=6,12,18,24,30$ respectively.

Condition LT2 The $a_{i}$ 's are constant up to relative position $1 / 2$, and linearly dependent on $i$ from this position onwards. In this case a difference of $\sigma$ between $a_{1}$ and $a_{n}$ corresponds to the value $.3849, .3544, .3440, .3387, .3355$ of $\Delta\left(a_{1} \ldots, a_{n}\right)$ for $n=6,12,18,24,30$ respectively.

Condition LT3 The $a_{i}$ 's are constant up to relative position $2 / 3$, and linearly dependent on $i$ from this position onwards. In this case a difference of $\sigma$ between $a_{1}$ and $a_{n}$ corresponds to the value $.3819, .3359, .3203, .3125, .3078$ of $\Delta\left(a_{1} \ldots, a_{n}\right)$ for $n=6,12,18,24,30$ respectively.

Condition SS2 The $a_{i}$ 's exhibit a sudden shift at relative position 1/2. Observe that a shift of $\sigma$ over the whole sample corresponds to the value .5000 of $\Delta\left(a_{1} \ldots, a_{n}\right)$.

Condition SS3 The $a_{i}$ 's exhibit a sudden shift at relative position $2 / 3$. Observe that a shift of $\sigma$ over the whole sample corresponds to the value 4714 of $\Delta\left(a_{1} \ldots, a_{n}\right)$.

Under each of the six out-of-control conditions we estimated the signalling probabilities of the charts in Figure 1. All charts were designed to have an overall in-control signalling probability equal to 0.05 .

Observe that in all Cusum charts we have taken $f$ equal to zero. This was done to allow fair comparison with the LRT chart. In a certain sense $f$ acts as a cut-off value: Cusum charts are insensitive to departures which are relatively small with respect to $f$. Only Cusum charts with $f$ set to zero share the ability of the LRT chart to detect all departures in the long run. Later in this section we shall comment on the effect of choosing a positive value of $f$ on the performance of Cusum charts. 

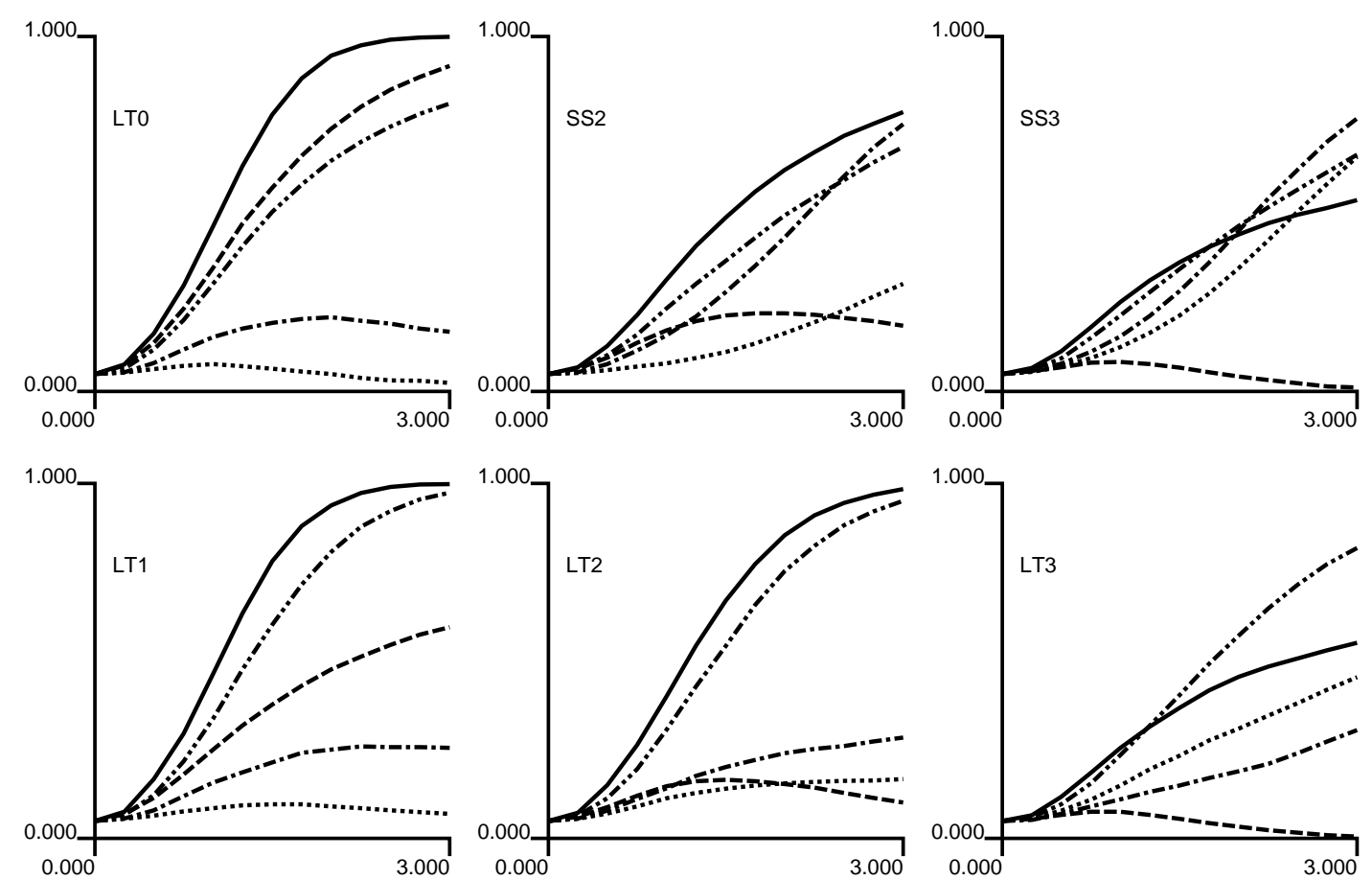

\section{Legend}

- proposed Cusum chart

- - BDE chart

-...' LRT chart

-.... Q-Cusum chart

....... Q-chart

Figure 2: Simulation results for sample size 6: signalling probability versus $\Delta\left(a_{1}, \ldots, a_{n}\right)$. On the upper row out-of-control conditions LT0, SS2, SS3, on the lower row out-of-control conditions LT1, LT2, LT3.

Figure 2 summarizes the simulation results for $n=6$ in six plots; every plot pertains to one of the out-of-control conditions LT0, SS2, SS3, LT1, LT2, LT3]. Striking in these plots is the so-called "masking effect", a consequence of using non-robust variance estimators. The masking effect is most prominently present in the behavior of the $Q$-chart under condition LT0, and in the behavior of the BDE Cusum chart under conditions SS2, LT2 and LT3: here the signalling probability clearly tends to zero as $\Delta\left(a_{1}, \ldots, a_{n}\right)$ grows large. To a lesser extent the masking effect shows up in the behavior of the LRT chart under condition LT0. The proposed Cusum chart and also the $Q$-Cusum chart seem less vulnerable.

For relatively small values of $\Delta\left(a_{1}, \ldots, a_{n}\right)$ [where the masking effect is virtually absent] the Cusum charts show the best performance, and the $Q$-chart the worst.

The simulation results for $n=12$ are summarized in the six plots of Figure 3. In all plots the proposed Cusum chart is clearly better than the other charts, at some distance followed by the $Q$-Cusum chart. The masking effect is still clearly present in the behavior of the BDE Cusum chart under conditions SS3, LT2 and LT3.

The simulation results for $n=18$, which are summarized in the six plots of Fig- 

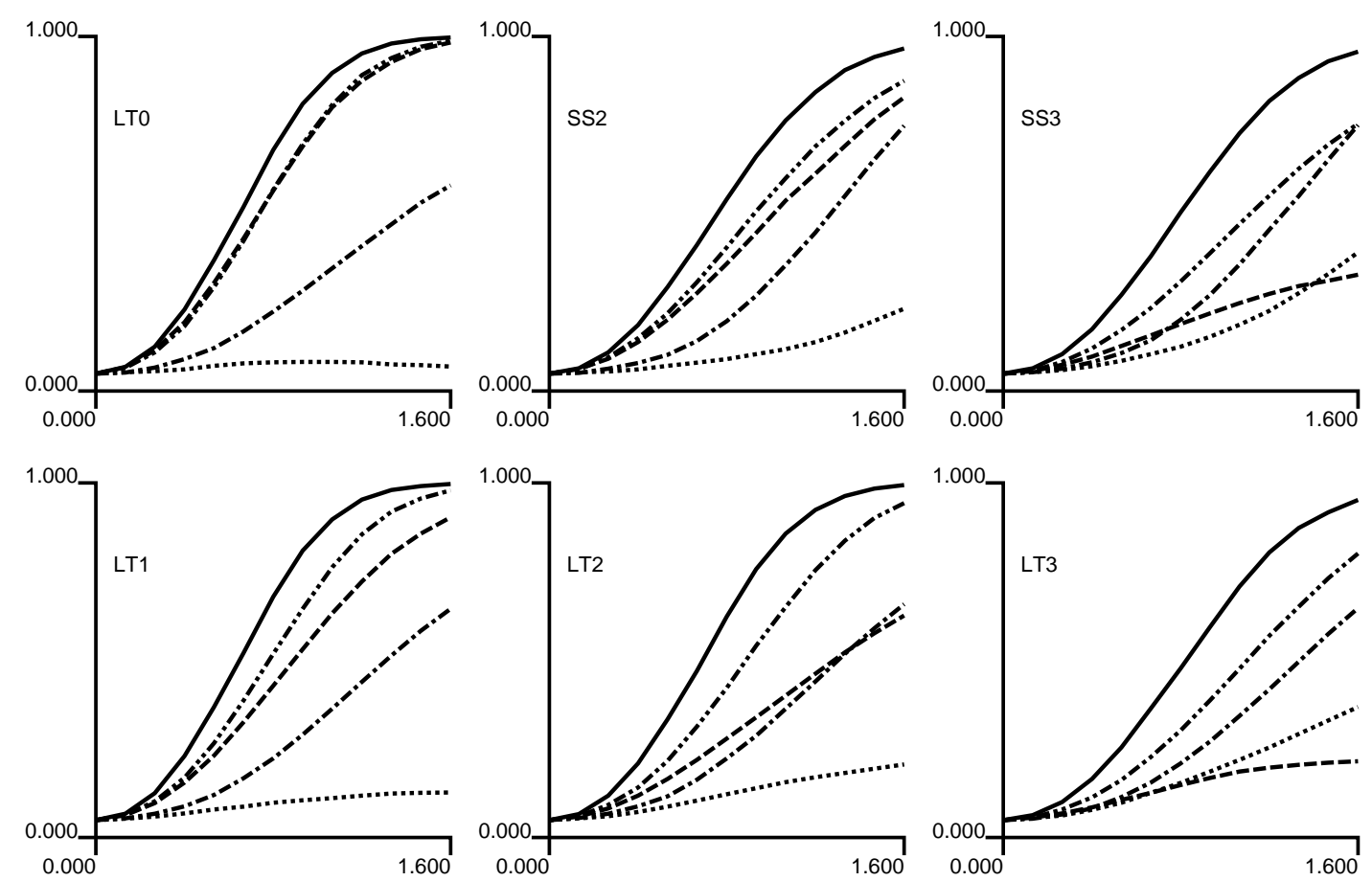

\section{Legend}

- proposed Cusum chart

- - BDE chart

-...' LRT chart

-.... Q-Cusum chart

....... Q-chart

Figure 3: Simulation results for sample size 12: signalling probability versus $\Delta\left(a_{1}, \ldots, a_{n}\right)$. On the upper row out-of-control conditions LT0, SS2, SS3, on the lower row out-of-control conditions LT1, LT2, LT3.

ure 4, roughly yield the same conclusion. However, the BDE Cusum chart appears to be recovering from the masking effect and starts to catch up with the $Q$-Cusum chart. For $n=24$ and $n=30$ the same conclusions hold.

Our simulation study did also include many variants of the three types of Cusum charts considered in this paper. Obviously, variants can be obtained by varying the value of $f$ [cf. (6)]. Except for small $n$, all Cusum charts with $f$ positive showed worse performance than their counterparts with $f$ equal to zero. Thus, a positive value of $f$ decreases the sensitivity of the chart, but may offer better protection to the masking effect.

Other variants are obtained by reversing the order in which the cumulation takes place, or by reversing the order in which the recursive residuals are computed. The four possibilities are given in Table 3. Although Schweder (1976) advocates the use of backward Cusums when the situation is initially in-control, we argue in Appendix A that there is no special advantage in using backward Cusums over forward Cusums. However, time-reversion does matter. For instance, it greatly improves the behavior of the BDE Cusum chart under conditions SS2, SS3, LT1, LT2 and LT3 for $n \geq 18$. It 

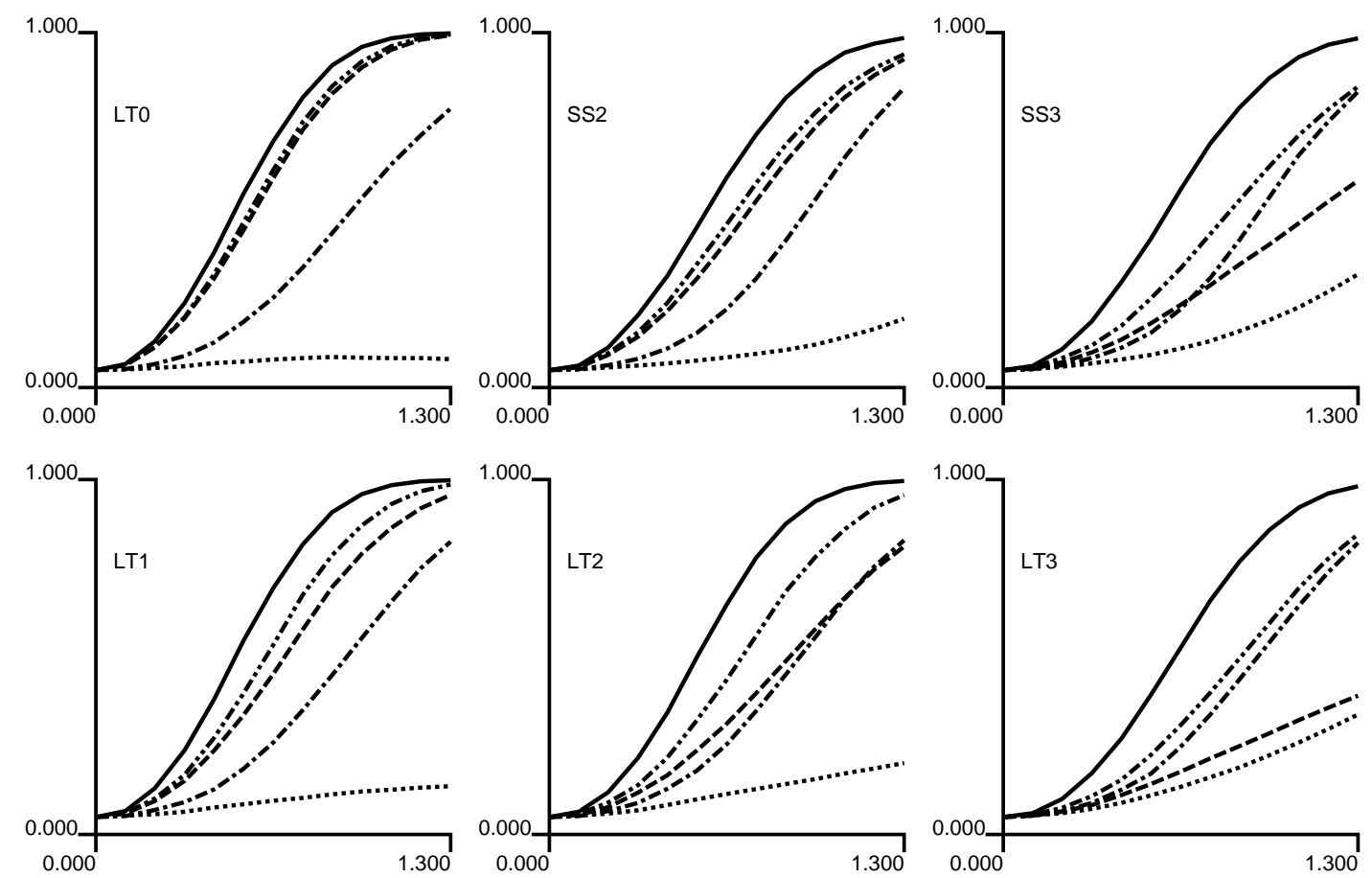

\section{Legend}

- proposed Cusum chart

- - BDE chart

-...' LRT chart

-.... Q-Cusum chart

....... Q-chart

Figure 4: Simulation results for sample size 18: signalling probability versus $\Delta\left(a_{1}, \ldots, a_{n}\right)$. On the upper row out-of-control conditions LT0, SS2, SS3, on the lower row out-of-control conditions LT1, LT2, LT3.

also improves the behavior of the $Q$-Cusum chart under conditions LT1, LT2 and LT3.

If we include the time-reversed versions in our comparisons, the time-reversed BDE Cusum chart is to preferred if the sample size is sufficiently large and out-ofcontrol conditions emerge relatively late [LT2, LT3]. It immediately follows that the "ordinary" BDE Cusum chart is to be preferred if the sample size is sufficiently large and out-of-control conditions emerge relatively early. Under all other circumstances the proposed Cusum chart shows the strongest performance.

\section{A Appendix: a generalized Cusum chart}

In this appendix we generalize the proposed Cusum chart. Suppose that instead of (5) the linear model

$$
X_{i}=\mu+c_{i} \theta+\epsilon_{i}, \quad i=1,2, \ldots
$$




\begin{tabular}{|c|cc|}
\hline $\begin{array}{c}\text { Order of computing } \\
\text { recursive residuals }\end{array}$ & \multicolumn{2}{|c|}{ Order of cumulation } \\
\hline not reversed & forward Cusum & backward Cusum \\
\hline reversed & time-reversed & time-reversed \\
& backward Cusum & forward Cusum \\
\hline
\end{tabular}

Table 3: Variants of Cusum charts obtained reversing the order of cumulation and/or the order of computing recursive residuals.

holds. As before, the $\epsilon_{i}$ 's are independent normal random variables with expectation zero and unknown variance $\sigma^{2}$. Under this model we may show that

$$
Y_{i}=d_{i} \theta+\delta_{i}
$$

where $\delta_{i}$ is as before, and

$$
d_{i}=\sqrt{\frac{i-1}{i}}\left(c_{i}-\frac{1}{i-1} \sum_{j=1}^{i-1} c_{j}\right) .
$$

The joint density of $Y_{2}, \ldots, Y_{i}$ has a monotone likelihood ratio in

$$
U_{i}=\sum_{j=2}^{i} d_{j} Y_{j}
$$

with respect to $\theta$. Thus, if $Y_{2}, \ldots, Y_{i}$ are observed [and $\sigma^{2}$ is known], then the onesided test based on $U_{i}$ is uniformly most powerful for $\theta$. One may show that $U_{i}$ is in fact the score statistic for this problem. Box and Ramirez (1992) proposed a score chart in a stage 2 context.

Observe that both the expectation and the variance of $U_{i}$ are linear in $\sum_{j=2}^{i} d_{j}^{2}$, which suggests applying a V-mask procedure to the plot of $U_{i}$ versus $\sum_{j=2}^{i} d_{j}^{2}$, or equivalently, imposing a control limit $h$ on the pair of one-sided cumulative sums $S_{H, i}$ and $S_{L, i}$ defined by

$$
\begin{gathered}
S_{H, i}=\max \left(0, S_{H, i-1}+d_{i}\left(Y_{i}-f d_{i}\right)\right), \\
S_{L, i}=\max \left(0, S_{L, i-1}+d_{i}\left(-Y_{i}-f d_{i}\right)\right) .
\end{gathered}
$$

We shall refer to $S_{H, i}$ and $S_{L, i}$ as the upper and lower Cusum, respectively.

Ideally, the one-sided cumulative sums $S_{H, i}$ and $S_{L, i}$ should be plotted versus $\sum_{j=2}^{i} d_{j}^{2}$.

In case $\sigma^{2}$ is unknown, the charts are readily adapted by plugging in the estimator $S_{n}^{2}$ as defined in (4). 
Observe that the BDE Cusum chart is in fact a special case of our chart, with $d_{i}$ 's being constant. Observe that in our derivation constant $d_{i}$ 's are obtained when the $c_{i}$ 's satisfy approximately

$$
c_{i} \approx c_{1}+\frac{1}{i-1} \sum_{j=1}^{i-1} c_{j} .
$$

Rewriting this expression yields

$$
c_{i} \approx c_{1}\left(1+\sum_{j=1}^{i-1} \frac{1}{j}\right) \approx c_{1}(1+\gamma+\log (i-1)),
$$

where $\gamma=0.5772 \cdots$ denotes Euler's constant. This suggests that the BDE Cusum chart is in particular strong in detecting out-of-control condition which show up relatively early in the sample.

Finally, consider a situation in which an in-control period is followed by an outof-control period. Since every $d_{j}$ belonging to the in-control period is equal to zero, it follows from the theory above that the uniformly most powerful one-sided test is based on a statistic of the form

$$
U_{i}^{*}=\sum_{j=i}^{n} d_{j} Y_{j}
$$

where $i$ denotes the start of the out-of-control period. At a first glance this seems to suggest that in this situation plotting $U_{i}^{*}$ versus $\sum_{j=i}^{n} d_{j}^{2}$ may be preferable over plotting $U_{i}$ versus $\sum_{j=2}^{i} d_{j}^{2}$. In the special case that every $d_{j}$ belonging to the out-ofcontrol period is equal to 1 , the chart based on $U_{i}^{*}$ reduces to the backward cumulative sum described in Schweder (1976) [cf. also Hinkley (1973)]. However, a closer look reveals that the signalling probability of the upper forward Cusum should coincide with the signalling probability of the lower backward Cusum. Similar statements hold true for the signalling probabilities of the lower forward Cusum and the upper backward Cusum, and the signalling probabilities of the two-sided forward Cusum and the twosided backward Cusum. Thus, there is no special advantage in using backward Cusums over forward Cusums.

\section{References}

[1] Barlow, R.E., Campo, R. (1975). "Total time on test processes and applications to failure data analysis". Reliability and Fault Tree Analysis, 451-481. SIAM, Philadelphia, Pennsylvania.

[2] Billingsley (1968). Convergence of Probability Measures. Wiley, New York.

[3] Box, G., Ramirez, J. (1992). "Cumulative score charts". Quality and Reliability Engineering International 8, 17-27. 
[4] Brown, R.L., Durbin, J., Evans, J.M. (1975). "Techniques for testing the constancy of regression relationships over time". Journal of the Royal Statistical Society B37, 149-163.

[5] Basu, D. (1955). "On statistics independent of a complete sufficient statistic". Sankhya 15, 377-380.

[6] Del Castillo, E., Montgomery, D.C. (1994). "Short-run statistical process control: Q-chart enhancements and alternative methods". Quality and Reliability Engineering International 10, 87-97.

[7] Hinkley, D.V. (1971). "Inference about the change-point from cumulative sum tests". Biometrika 58, 509-523.

[8] Lehmann, E.L. (1994). Testing Statistical Hypotheses. Second edition Chapman and Hall, London.

[9] Lucas (1982). "Combined Shewhart-CUSUM quality control schemes". Journal of Quality Technology 14, 51-59.

[10] Montgomery, D.C. (1996). Introduction to Statistical Quality Control, third edition. Wiley, New York.

[11] Nelson, L.S. (1984). "The Shewhart control chart - Tests for special causes". Journal of Quality Technology 16, 237-239.

[12] Page, E.S. (1954). “Continuous inspection schemes”. Biometrika 41, 100-115.

[13] Quandt, R.E. (1960). "Tests of the hypothesis that a linear regression system obeys two seperate regimes". Journal of the American Statistical Association 55, 324-330.

[14] Quesenberry, C.P. (1991). "SPC $Q$ charts for start-up processes and short or long runs". Journal of Quality Technology 23, 213-224.

[15] Quesenberry, C.P. (1995). “On properties of Q charts for variables”. Journal of Quality Technology 27, 184-203.

[16] Schweder, T. (1976). "Some "optimal" methods to detect structural shift or outliers in regression". Journal of the American Statistical Association 71, 491-501.

[17] Shewhart, W.A. (1931). Economic Control of Quality of Manufactured Product. Van Nostrand Reinhold Company, Princeton.

[18] Sullivan, J.H., Woodall, W.H. (1996). "A control chart for preliminary analysis of individual observations". Journal of Quality Technology 28, 265-278.

Key Words: Cusum chart, control chart, statistical process control. 